\title{
Lidil
}

Revue de linguistique et de didactique des langues

Multimodalité de la communication chez l'enfant

\section{La gestualité cohésive dans les récits d'enfants âgés de 9 à 11 ans}

\section{Djaber Fantazi}

\section{OpenEdition}

\section{Journals}

Édition électronique

URL : http://journals.openedition.org/lidil/3074

DOI : 10.4000/lidil.3074

ISSN : $1960-6052$

\section{Éditeur}

UGA Éditions/Université Grenoble Alpes

\section{Édition imprimée}

Date de publication : 30 novembre 2010

Pagination : $97-112$

ISBN : $978-2-84310-184-7$

ISSN : 1146-6480

\section{Référence électronique}

Djaber Fantazi, «La gestualité cohésive dans les récits d'enfants âgés de 9 à 11 ans », Lidil [En ligne], 42 | 2010, mis en ligne le 31 mai 2012, consulté le 01 mai 2019. URL : http://journals.openedition.org/ lidil/3074; DOl : 10.4000/lidil.3074 


\title{
La gestualité cohésive dans les récits d'enfants âgés de 9 à 11 ans
}

\author{
Djaber Fantazi*
}

\begin{abstract}
RÉSUMÉ
Cette étude montre, à partir de l'analyse de récits enfantins, que le geste participe, au même titre que la parole, à la cohésion discursive. Nous avons observé un corpus audiovisuel de 31 récits oraux produits par des élèves de cours moyen d'une école de l'agglomération grenobloise. Nos résultats montrent que parmi les gestes accompagnant la parole, certains peuvent effectivement remplir une fonction cohésive dès lors qu'ils établissent une relation de connexité (démarcative) ou de thématisation (référentielle) avec des structures langagières produites antérieurement. Bien que ce résultat soit obtenu dans le langage oral, ses conséquences didactiques peuvent s'étendre au langage écrit, puisque la gestualité cohésive, au-delà de son aspect kinésique restreint, renseigne sur la capacité du locuteur à produire du langage organisé en discours.
\end{abstract}

\section{ABSTRACT}

This analysis of children's narratives shows that gesture contributes, as well as speech, to textual discourse cohesion. We studied an audiovisual corpus of 31 oral narratives produced by pupils of a primary school situated in Grenoble, France. Our results showed that among the co-speech gestures, some may actually serve a cohesive function when they fulfill a connectivity relationship (demarcation) or a referential introduction with language structures previously mentioned. Although this result is obtained in oral language, it may also extend to teaching written language since the cohesive gesture, beyond its limited kinetic aspect, enables the speaker to produce organized discourse.

* LIDILEM, Université de Grenoble. 


\section{La gestualité cohésive dans les récits enfantins}

Longtemps centrée sur les aspects verbaux de la communication, l'étude du langage oral intègre de plus en plus les aspects non verbaux à mesure que l'analyse de la production langagière nécessite, outre les dimensions énonciatives et linguistiques, les dimensions pragmatiques et discursives. Ainsi, l'étude du non-verbal s'est considérablement affinée ces dernières années, notamment pour ce qui est des gestes à visée communicative comme les coverbaux. Ceux-ci peuvent être définis comme des mouvements corporels produits par le locuteur en accompagnement de sa parole. Ils sont orientés soit vers l'échange discursif dans les situations dialogales soit vers l'élaboration du texte dans les situations monologales. Les coverbaux ont fait l'objet de nombreuses études chez l'enfant comme chez l'adulte, en France (Cosnier et Brossard, 1984; Calbris, 2002; Colletta, 2004; Colletta, Pellenq et Guidetti, 2010), comme à l'étranger (Kendon, 1972; McNeill, 1992, 2000 ; Kita et Özyürek, 2007; Gullberg, de Bot et Volterra, 2008). Ainsi, toutes ces études s'accordent à dire que les coverbaux font partie intégrante du système de la parole et ne peuvent être interprétés sans celui-ci. Cela dit, des tentatives de catégorisation des coverbaux (Scherer, 1984; McNeill, 1992; Cosnier et Vaysse, 1997; Colletta, 2004) ont vu le jour, dont le but principal est d'établir les fonctions que ces gestes remplissent dans la communication à l'oral. Parmi les fonctions qui font l'unanimité des gestualistes, on trouve la fonction référentielle, la fonction de cadrage, la fonction de structuration et la fonction interactive (Calbris, 1997). En plus de ces quatre fonctions, on peut en envisager une cinquième, mise au jour par McNeill (1992), il s'agit de la fonction cohésive, qui, à la différence des précédentes qui se manifestent au niveau de l'énoncé, se réalise, elle, au niveau textuel. Selon McNeill, elle se matérialise à travers des reprises ou anaphores gestuelles, ou encore, des beats ou gestes de battement accompagnant des connecteurs.

Dans le cadre cette étude, nous accordons une attention toute particulière à la fonction cohésive des coverbaux enfantins, cela pour deux raisons : d'abord, les gestes cohésifs assument d'autres fonctions (référentielle et de structuration) au niveau de l'énoncé qui sont intéressantes à analyser; ensuite, cette fonction cohésive, loin de n'être que le reflet des habilités kinésiques du locuteur orchestrées ou non avec la parole, renseigne sur une capacité plus générale qui est la capacité à produire du langage organisé en discours. Si l'adulte arrive aisément à 
produire du langage organisé en discours, ce n'est pas toujours le cas pour le jeune enfant.

Des études développementales et multimodales récentes (Colletta, Pellenq et Guidetti, 2010) montrent que le discours enfantin évolue avec l'âge, sur le plan verbal comme sur le plan non verbal. Au niveau verbal puisque l'acquisition des outils textuels se complexifie avec l'âge, et au niveau non verbal puisque les coverbaux enfantins loin de régresser, se transforment et continuent à se développer avec l'âge tout au long de l'enfance (Colletta, 2004; Gullberg, de Bot et Volterra, 2008). Ils passent d'un usage simple : déictique, à un usage beaucoup plus diversifié : iconique et référentiel via l'emploi de gestes métaphoriques et de désignation endophorique (McNeill, 1992; Colletta, 2004). Toutefois, si le discours enfantin évolue avec l'âge, la capacité à produire du langage organisé en discours (texte cohésif) évolue bien lentement et ne se met pas en place avant l'âge de 9-10 ans. À partir de cet âge, le langage enfantin connait une véritable explosion; l'enfant devient capable de construire des textes cohésifs et cohérents comme ceux de l'adulte. Ici, nous pouvons nous demander, en vertu de la thèse de la multimodalité de la parole (Kendon, 1972; McNeill, 1992), ce qu'il en est de la gestualité cohésive à cet âge seuil.

Dans cet article nous examinons les coverbaux enfantins en matière de cohésion narrative, en visant une tranche d'âge bien spécifique qui est la fin de la scolarité primaire, là où l'enfant devient capable de produire des récits complets. Le premier objectif de cette étude vise, à la lumière des observations de McNeill (1992), à compléter le travail de Colletta, Pellenq et Guidetti (2010) sur l'emploi (diversité et ampleur) des gestes participant à la cohésion discursive. Le second objectif consiste en une exploration de la gestualité cohésive afin de délimiter la part dévolue au produit de la narration (la construction référentielle) et celle dévolue à la gestion de ce produit (la structuration de l'activité narrative). Dans un premier temps, nous présentons les fonctions des coverbaux les plus représentées dans le langage narratif d'élèves de fin de cycle trois. Ensuite, nous mettrons en relief la répartition des gestes cohésifs selon leurs classes d'appartenance. Enfin, des études de cas tirées de notre corpus permettront de montrer la double fonction (énoncé-texte) du geste cohésif. Mais pour l'heure, voyons d'abord les éléments méthodologiques qui ont guidé l'étude. 


\section{Méthodologie}

Le corpus a été recueilli dans une école primaire de l'agglomération de Grenoble; il est constitué de 31 récits oraux produits par une population d'enfants âgés de 9 à 11 ans et scolarisés en CM1 et CM2 (fin de scolarité primaire). Le protocole de recueil de données, que nous utilisons dans cette étude, a été conçu par Colletta et ses collaborateurs en 2005 à l'occasion du projet ANR0178-08 «Multimodalité». Ce protocole comporte deux étapes : d'abord, chaque enfant passe un test d'évaluation du langage oral ELO. Ce test s'administre dans le but de contrôler les capacités lexicales et syntaxiques de l'enfant et de disposer ainsi d'une mesure précise permettant d'écarter de l'étude les enfants en retard langagier.

Le tableau 1 ci-après indique, entre autres caractéristiques, l'âge ${ }^{1}$ moyen des enfants.

\begin{tabular}{|l|c|c|}
\hline Dessin animé & RT\&J & RW\&G \\
\hline Âge moyen (an, mois) & 9 ans & 10 ans $10 \mathrm{~ms}$ \\
\hline Âge (min-max) & 8 ans $10 \mathrm{~ms}-10$ ans & 9 ans $11 \mathrm{~ms}-11$ ans $4 \mathrm{~ms}$ \\
\hline Effectif & 19 & 12 \\
\hline Filles /garçons & $10 / 9$ & $9 / 3$ \\
\hline
\end{tabular}

Tableau 1. - Caractéristiques de la population d'étude.

La deuxième étape consiste en un visionnement d'un clip vidéo de moins de $3 \mathrm{mn}$ (extrait d'un épisode de Tom et Jerry pour les uns, extrait d'une aventure de Wallace et Gromit pour les autres) ${ }^{2}$ présentant une mini-histoire complète avec début et fin. Ensuite, chaque enfant produit

1. Ici, l'âge est renseigné de façon indicative et n'est pas pris en compte comme variable car les capacités discursives évoluent lentement entre 9 et 11 ans et la plupart des chercheurs en psychologie du développement langagier préfèrent grouper les enfants par tranches d'âge.

2. Le choix d'utiliser deux vidéos différentes s'inscrit dans le cadre général de notre thèse. Nous avons montré ailleurs (Fantazi et Colletta, 2010) que ces deux vidéos (récit d'action vs récit de situation), génèrent, lors du rappel de récit, des gestes différents en particulier pour les iconiques. Dans le cas présent, l'objectif n'est pas d'explorer l'effet du contenu narratif, mais simplement de décrire et comprendre la gestualité enfantine dans sa plurifonctionnalité. 
un récit oral monologal en présence d'un interlocuteur. Si ce dernier a reçu la consigne de ne pas influencer la production de l'enfant, il pourra en revanche relancer le récit en cas de silence. Pour obtenir un texte «narratif», la consigne adressée à l'enfant est de raconter l'histoire qu'il vient de regarder. Pendant que l'enfant raconte l'histoire, un caméscope enregistre son activité verbo-gestuelle.

Les récits filmés ont été par la suite transcrits et codés sous les dimensions syntaxique, narrative et gestuelle à l'aide du logiciel ELAN, qui grâce à l'alignement de la transcription sur les sources audio et vidéo, permet un repérage précis du message verbal.

Pour ce qui est des faits gestuels dans les récits, ceux-ci ont été catégorisés selon la grille de Colletta et ses collaborateurs, (Colletta, Kunene, Venouil, Kaufmann et Simon, 2009), et qui apparait la plus adaptée à notre étude. Cette grille comporte sept catégories de gestes, (ci-dessous chaque catégorie est accompagnée d'exemple(s) tiré(s) de notre corpus), on y trouve :

1. Le déictique : geste manuel ou céphalique de pointage dirigé vers un objet présent dans la situation, vers l'interlocuteur, vers soi-même...

Ex. : le locuteur s'auto-désigne en disant «c'est ce que moi j'ai compris».

2. Le représentationnel ou le référentiel : geste manuel ou mimique faciale, associant ou non d'autres parties du corps, qui représente un objet concret ou qui symbolise une idée abstraite. Il peut être :

- illustratif; ex. : deux mains dessinant la forme d'un contenant pour représenter un nid;

- locatif; ex. : geste manuel de pointage, localisant un personnage dans l'espace frontal;

- mimétique; ex. : expression faciale de perplexité pour représenter un personnage qui ne trouve pas de solution et s'interroge;

- figuratif; ex. : geste métaphorique représentant un concept abstrait (l'histoire, le temps, une vertu, etc.);

- anaphorique; ex. : pointage manuel abstrait vers une zone de l'espace frontal préalablement assignée à un personnage, pour le représenter une deuxième fois;

- substitutif; ex. : pointage manuel désignant dans la situation un référent venant se substituer au référent du discours (p.ex. 
pointer vers un meuble pour représenter la commode d'une histoire).

3. Le geste de cadrage : geste réalisé notamment dans la narration et qui exprime un état émotionnel ou un état mental du locuteur. Ex. : visage prenant les traits de l'amusement pour exprimer le côté comique d'une situation qu'on rapporte.

4. Le geste de structuration ou discursif : geste généralement bref qui structure la parole ou le discours par l'accentuation ou la mise en relief de certaines unités linguistiques ou autres. Il peut être :

- démarcatif; ex. : mouvement rapide de la main esquissant le geste de chasser quelque chose pour signifier que le locuteur revient au récit après un commentaire;

- rythmique; ex. : succession de mouvements rythmiques de la main pour segmenter la phrase suivante : [...] après Jerry :: $/ /$ eh elle croit eh [...];

- intensif; ex. : mouvement intensif de la tête pour accentuer le mot DÉTRUIRE la commode.

5. Le geste interactif : comme son nom l'indique, il s'agit d'un geste et/ou regard par lequel le locuteur vérifie l'attention de son interlocuteur ou l'approche de son tour de parole.

Ex. : hochement de la tête pendant que l'interlocuteur parle.

6. Le geste énonciatif : geste manuel ou expression faciale qui manifeste que le locuteur cherche un mot ou une expression.

Ex. : froncement de sourcils et regard vers le haut tout en cherchant ses mots.

7. Le geste performatif : geste qui permet la réalisation non verbale d'un acte de langage non assertif (réponse, question...), ou qui renforce ou modifie la force illocutoire lorsqu'il est verbalisé. Ex. : hochement de tête en guise de réponse affirmative.

Comme la gestualité cohésive puise ses ressources dans les gestes référentiels et de structuration, nous avons tenu compte dans l'observation des faits gestuels, non seulement des gestes manuels, mais aussi des gestes céphaliques et des changements de postures. Afin de disposer de valeurs et formes objectives des gestes, plusieurs annotateurs, dont un juge, tous préalablement formés à l'annotation gestuelle, ont été sollicités. 


\section{Résultats et discussion}

Une première exploration des coverbaux consiste à les dénombrer. Le nombre total s'élève à 469 gestes coverbaux dans le corpus. Comme la variabilité inter-sujets dans la production verbo-gestuelle est importante, nous avons mesuré le taux gestuel ${ }^{3}$. Il s'avère qu'en moyenne l'élève de fin d'école primaire produit un coverbal toutes les trois propositions.

\begin{tabular}{|l|c|}
\hline Nombre moyen de propositions & 46 \\
\hline Nombre moyen de gestes & 14,27 \\
\hline Taux gestuel moyen (écart-type) & $0,31(0,19)$ \\
\hline
\end{tabular}

Tableau 2. - Taux gestuel moyen (avec écart type).

Une deuxième exploration consiste à dénombrer les coverbaux selon les fonctions qu'ils remplissent dans le récit. Ces fonctions sont rapportées dans le tableau 3 ci-dessous.

\begin{tabular}{|l|c|}
\hline Fonctions des gestes & nombre de gestes (avec pourcentage) \\
\hline Gestes référentiels & $260(52,96 \%)$ \\
\hline Gestes de cadrage & $99(22,96 \%)$ \\
\hline Gestes de structuration & $57(13,33 \%)$ \\
\hline Gestes interactifs & $18(3,12 \%)$ \\
\hline $\begin{array}{l}\text { Autres gestes : } \\
\text { (énonciatif, performatif...) }\end{array}$ & $35(7,61 \%)$ \\
\hline Total & $\mathbf{4 6 9}(100 \%)$ \\
\hline
\end{tabular}

Tableau 3. - Valeur des gestes dans le corpus.

3. Étant donné que la longueur des récits varie de façon importante d'un élève à un autre, nous avons été amené à comparer la production gestuelle en tenant compte de la production verbale. Le moyen le plus communément utilisé est de diviser le nombre de gestes par le nombre de propositions pour obtenir un «taux gestuel».

Il existe d'autres méthodes de calcul comme le nombre de gestes par seconde ou par minute. Nous avons exclu l'emploi de celles-ci dans notre étude puisque la durée d'un récit est faite de paroles, mais aussi de pauses et de silences, ce qui rend le calcul du taux gestuel moins précis. En revanche, la proposition a été adoptée comme la base de calcul du taux gestuel en vertu de ses relations fonctionnelles, séquentielles et sémiotiques privilégiées à l'égard des unités gestuelles (McNeill, 1992, 2000; Kita et Özyürek, 2007). 
Ce tableau montre que les gestes référentiels et de cadrage sont les plus nombreux dans la narration enfantine à l'oral au cours moyen. Cette première constatation va dans le sens des résultats trouvés sur l'ensemble du corpus ANR (Colletta, Pellenq et Guidetti, 2010), beaucoup plus vaste $(\mathrm{N}=122$, dont 84 enfants), où il apparait que les gestes référentiels et de cadrage jouent un rôle substantiel dans les récits enfantins. Les référentiels aident à la représentation des évènements et des actions constituant la trame évènementielle du récit, tandis que les gestes de cadrage expriment les intentions du locuteur au cours de l'activité narrative.

\section{Les gestes cohésifs}

Enfin, une exploration d'ordre qualitatif consiste à étudier l'emploi de la gestualité coverbale au cours de la production du récit. Celle-ci nous a conduit à déceler la double fonction de certains gestes de la catégorie des référentiels et des gestes de structuration. Dans les lignes qui suivent, nous montrons comment ces gestes remplissent des fonctions différentes selon qu'ils sont saisis au niveau de l'énoncé ou au niveau du texte. En effet, un geste de structuration, tel qu'un démarcatif au niveau de l'énoncé, peut devenir cohésif au niveau textuel en marquant une rupture au cours de l'activité narrative ou une transition de la narration vers le commentaire. De même, un geste référentiel au niveau de l'énoncé peut, par reprise d'un référent antérieur, devenir un cohésif au niveau textuel grâce à l'iconicité et/ou au pointage à valeur anaphorique. Mais avant cela, voyons d'abord la répartition de ces gestes en fonction du niveau de réalisation.

\section{Répartition des gestes cohésifs}

Comme le montre le tableau 4, les gestes plurifonctionnels ou cohésifs les plus représentés sont dans l'ordre les iconiques illustratifs suivis par les abstraits anaphoriques avec respectivement 35 et $28 \%$. Pour les gestes plurifonctionnels au service de la structuration, ce sont les intensifs et les démarcatifs avec pareillement $7 \%$ chacun de la totalité des gestes cohésifs. 


\begin{tabular}{|c|c|c|c|c|}
\hline & & & \multicolumn{2}{|c|}{$\begin{array}{c}\text { Niveaux de réalisation } \\
\text { (local, global) }\end{array}$} \\
\hline & & & $\begin{array}{l}\text { Gestes pluri- } \\
\text { fonctionnels }{ }^{4} \\
\text { (énoncé-texte) } \\
\text { Nombre de gestes } \\
\text { (avec \%) }\end{array}$ & $\begin{array}{l}\text { Gestes uni- } \\
\text { fonctionnels } \\
\text { (énoncé) } \\
\text { Nombre de gestes } \\
\text { (avec \%) }\end{array}$ \\
\hline \multirow{3}{*}{\multicolumn{2}{|c|}{$\begin{array}{l}\text { Gestes de structuration } \\
\text { (appelés aussi discursifs) }\end{array}$}} & Intensif & $4(7 \%)$ & $18(11,61 \%)$ \\
\hline & & Rythmique & $0(0 \%)$ & $5(3,22 \%)$ \\
\hline & & Démarcatif & $4(7 \%)$ & $8(5,16 \%)$ \\
\hline \multirow{7}{*}{$\begin{array}{l}\text { Gestes } \\
\text { référentiels }\end{array}$} & \multirow{4}{*}{ Iconiques } & Déictique & $2(3,57 \%)$ & $7(4,51 \%)$ \\
\hline & & Illustratif & $20(35 \%)$ & $58(37,41 \%)$ \\
\hline & & Locatif & $0(0 \%)$ & $12(7,74 \%)$ \\
\hline & & Mimétique & $2(3,57 \%)$ & $7(4,51 \%)$ \\
\hline & \multirow{3}{*}{ Abstraits } & Figuratif & $4(7 \%)$ & $8(5,16 \%)$ \\
\hline & & Anaphorique & $16(28 \%)$ & $26(16,77 \%)$ \\
\hline & & Substitutif & $1(1,78 \%)$ & $6(3,87 \%)$ \\
\hline \multicolumn{3}{|c|}{ Total } & $56(100 \%)$ & $155(100 \%)$ \\
\hline
\end{tabular}

Tableau 4. - Répartition du nombre ${ }^{5}$ (avec pourcentage) des coverbaux référentiels et de structuration selon leurs sous-classes et leurs niveaux de réalisation.

\section{Moments d'apparition des gestes cohésifs}

L'analyse du corpus fait apparaitre que les gestes référentiels et de structuration ne sont pas tous cohésifs. En effet, $64 \%$ des gestes remplissent leurs fonctions référentielle et de structuration au niveau de l'énoncé. L'examen minutieux des récits enfantins révèle deux situations aux cours desquelles les gestes de structuration et référentiels sont unifonctionnels. La première situation réfère à une production gestuelle en début de récit (là où les chaines anaphoriques commencent à peine

4. Tout geste plurifonctionnel est forcément réalisé au niveau local (énoncé) en même temps qu'au niveau global (texte), ce qui n'est pas le cas du geste uni-fonctionnel.

5. Vu le nombre important de gestes à observer : 469, nous avons volontairement réduit l'étude qualitative de la gestualité cohésive à la moitié du corpus : 234 gestes correspondant à 15 récits. Ces récits ont été choisis sur la base du nombre plus ou moins proche de gestes. 
à se former, lorsque quand l'enfant narrateur n'envisage pas encore de segmenter son discours); la seconde à une production gestuelle au cours du récit. Dans le cas des référentiels, à chaque fois que l'enfant introduit un nouvel objet narratif, correspondant à un référent que l'enfant juge vraisemblablement secondaire par rapport aux protagonistes ou aux évènements centraux, et qui ne mérite pas, à ses yeux, d'être relié aux référents thématiques : la stratégie de l'enfant consiste alors à isoler certains référents et à les maintenir au niveau local, c'est-àdire au niveau syntagmatique ou phrastique. Dans le cas des démarcatifs, ces gestes unifonctionnels se produisent chaque fois que l'enfant introduit un nouvel énoncé ou accentue des syllabes ou des unités lexicales voisines.

À l'inverse, 36\% des gestes démarcatifs et référentiels remplissent une double fonction (énoncé et texte) et se réalisent à la fois au niveau local et global de la narration. L'observation des faits gestuels dans les récits enfantins nous révèle que ce sont des gestes qui ne peuvent être réalisés pendant la présentation du récit ou la description de l'arrière plan de l'histoire, sans doute à cause de la rareté des reprises anaphoriques. En tout cas, ce sont des gestes qui évoquent un référent déjà exprimé par un autre geste antérieurement et assurent ainsi la continuité référentielle : ici, la stratégie de l'enfant consiste à relier le référent au niveau global, c'est-à-dire au niveau textuel ou thématique. Ces gestes à double fonction peuvent aussi se produire au cours du récit lorsqu'il y a une rupture, ou chute selon la terminologie labovienne (Labov, 1978), dans l'activité narrative, c'est-à-dire quand l'enfant décide de faire une transition d'ordre pragmatique : quand il passe du récit au commentaire ou l'inverse.

Les gestes cohésifs sont massivement cités de façon linguistique. Cela sous-tend, en tout cas, l'idée que les gestes cohésifs sont réalisés de deux manières : soit le geste est produit conjointement avec la parole (synchrone), c'est le cas le plus fréquent; soit il est produit en l'absence de celle-ci (asynchrone) ${ }^{6}$. Mais ce deuxième cas, rare, avec 3\% des gestes cohésifs rencontrés, est le plus intéressant et montre la plus value du geste dans la production de la parole, il consolide ainsi la thèse de la multimodalité de la communication parlée. Sinon, dans la plupart des cas des gestes cohésifs (synchrones), c'est la parole qui devance le geste. Une parole qui peut être une anaphore ou un substitut anapho-

6. Dans ce cas-là, il n'est pas considéré comme un emblème, c'est bien un coverbal produit de façon différée avec la parole. 
rique, dans le cas des cohésifs référentiels ou un connecteur chronologique du type «et puis», «ensuite», «après» dans le cas des cohésifs à valeur de structuration.

\section{En quoi consistent-ils?}

Commençons par les gestes de structuration, nous avons noté (voir le tableau 4 ci-avant) l'emploi de gestes démarcatifs et intensifs. Alors De quoi s'agit-il au juste? En ce qui concerne le démarcatif, celui-ci prend généralement la forme d'une modification posturale ou d'un changement de la direction du regard. Il peut s'agir aussi de mouvements de la main dessinant une forme de contenant pour symboliser le thème de l'histoire ou un épisode, ou encore de gestes rythmiques ou beats (brefs gestes manuels produits conjointement avec un connecteur). Enfin, un autre cas de figure peut concerner des mouvements de la main plus rapides qui délimitent ou segmentent le discours grâce à une rupture dans l'activité narrative ou à un changement d'épisode ou de plan. Le geste de Suzie (élève de CM2) dans l'extrait suivant l'illustre parfaitement :

Suzie :
$[\ldots]$ et après il y a quelqu'un qui téléphone et voilà \{rire\}
S. démarcatif
Battement de la tête et changement de posture de Av.>Ar. marquant la fin du récit
Info suppl.

Dans cet extrait du récit de Suzie, le geste de structuration consiste en un battement de la tête de l'avant vers l'arrière sur un connecteur dont le rôle ici est le bornage au niveau de l'énoncé «et voilà». En même temps, ce même geste marque une rupture dans l'activité narrative puisqu'il permet à l'enfant-narrateur de marquer une chute ou coda; retour à la conversation : «c'est fini» en parlant du récit. Ce qui permet au final une segmentation au niveau global (textuel) puisque ce geste de retrait postural métaphorise, d'une certaine manière, le retrait de l'activité narrative, en une dés-implication de la narration (raconter $v s$ finir de raconter). C'est ce qu'on appelle un décrochage à l'extérieur du récit : l'enfant marque une rupture dans l'activité narrative en faisant une transition d'ordre pragmatique.

Quant à l'intensif, c'est un geste qui souligne ou met en évidence une unité linguistique, son apport au niveau global est visible dans certains mouvements de la main ou de la tête accompagnant l'accentuation 
de certains mots (syllabe, unité lexicale, connecteurs...). L'extrait suivant du récit d'Anne (élève de CM1) montre comment le geste cohésif peut segmenter le discours narratif :

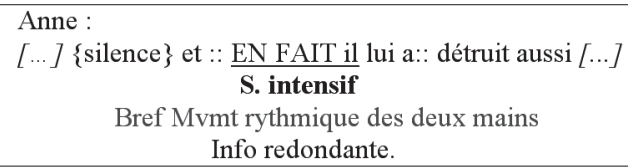

Dans cet extrait, le mouvement rythmique des deux mains vient accentuer les mots : «en fait...». Cette mise en relief d'unités linguistiques est accompagnée d'une légère modification prosodique au niveau de l'énoncé. Au niveau textuel, ce geste intensif joue un rôle démarcatif puisqu'il se produit après un silence et en accompagnement d'un connecteur, ce qui fait que ce geste contribue à la cohésion discursive au niveau global en segmentant le récit en unités aisément repérables et relativement autonomes.

Tout comme les gestes de structuration, les gestes référentiels à double fonction (énoncé-texte), comportent eux aussi deux grandes catégories : les gestes du concret dont la fonction est l'illustration, et les gestes de l'abstrait qui symbolisent des notions, des valeurs et des relations abstraites. Commençons par les plus nombreux dans le corpus : les gestes du concret et à leur tête les illustratifs. Ceux-ci représentent des caractéristiques physiques : dimensions, formes, aspects extérieurs, texture, etc., d'objets animés ou non. Concrètement, il s'agit de mouvements de la main qui représentent des référents concrets; ils sont iconiques à valeur anaphorique lorsque l'enfant répète un geste représentant un référent antérieur (personnage, objet, lieu...). La première séquence de l'extrait suivant du récit de Jonas (élève de CM1) fait surgir la double fonction de l'illustration iconique.

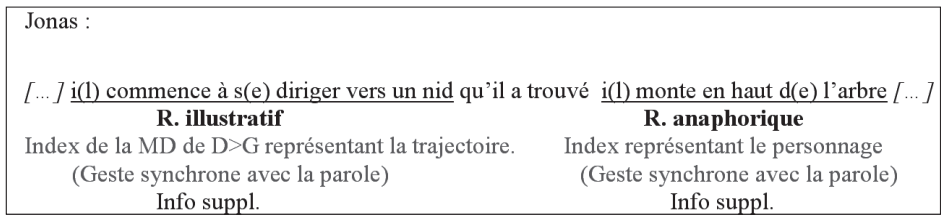

Dans cette séquence de récit, le geste iconique illustratif opère au niveau de l'énoncé puisqu'il représente une caractéristique physique du personnage : le déplacement de la souris; l'enfant reproduit la trajectoire de l'action de droite à gauche, typiquement comme dans le dessin 
animé. Ce geste illustratif opère aussi au niveau textuel grâce à son iconicité, où l'on décèle assez aisément la valeur anaphorique attribuée à l'index de la main droite pour représenter une propriété du référent : le déplacement de la souris.

La deuxième catégorie de gestes référentiels est celle des gestes de l'abstrait. Cette catégorie regroupe des gestes qui symbolisent des référents abstraits, par métaphore ou métonymie. Mais elle regroupe aussi des gestes de pointage abstrait, manuel ou céphalique, qui pointent un endroit de l'espace précédemment assigné à un personnage, comme dans la deuxième séquence de geste de Jonas, où la relation sémiotique entre le geste et le concept est de nature anaphorique, associant la main droite au référent «souris» au niveau de l'énoncé. Quant à la dimension textuelle de ce geste, elle est avérée grâce à la main droite qui désigne un endroit précis dans l'espace précédemment assigné au personnage de la souris.

En substance, les gestes cohésifs repérables au niveau textuel sont au moins de deux genres : $1^{\circ}$ utilisant des outils comme les anaphores ou leurs substituts pour représenter la continuité référentielle et thématique, ou $2^{\circ}$ utilisant des marques de connexité (connecteurs et ponctuants) pour segmenter le discours ou introduire des transitions du genre commentaire-narration (voir le tableau 5 ci-après [adapté de Colletta, 2004], présentant l'emploi des gestes référentiels et de structuration au niveau textuel ainsi que les moyens verbaux et gestuels déployés en vue de produire un discours cohésif).

\begin{tabular}{|l|l|l|l|l|}
\cline { 3 - 5 } \multicolumn{1}{c|}{} & Domaines & Fonctions & \multicolumn{2}{l|}{ Outils de réalisation au niveau textuel } \\
\hline \multirow{2}{*}{$\begin{array}{l}\text { Intégration } \\
\text { Cohésion } \\
\text { du discours } \\
\text { multimodal }\end{array}$} & $\begin{array}{l}\text { Assurer la } \\
\text { continuité } \\
\text { référentielle } \\
\text { et thématique }\end{array}$ & Linguistiques & $\begin{array}{l}\text { Reprises anaphoriques } \\
\text { et/ou leurs substituts, } \\
\text { isotopies sémantiques et } \\
\text { temporelles, marques de } \\
\text { connexité. }\end{array}$ \\
\cline { 3 - 5 } & Segmentation & $\begin{array}{l}\text { Démarquer } \\
\text { les unités qui } \\
\text { composent le } \\
\text { discours }\end{array}$ & Linguistiques & $\begin{array}{l}\text { Marques de connexité } \\
\text { (connecteurs ou } \\
\text { ponctuants prosodiques) }\end{array}$ \\
\cline { 3 - 5 } & & & $\begin{array}{l}\text { Gestes iconiques et/ou } \\
\text { de pointage à valeur } \\
\text { anaphorique }\end{array}$ \\
\cline { 3 - 5 } & & & Gestuels & $\begin{array}{l}\text { Gestes figuratifs ou } \\
\text { rythmiques }\end{array}$ \\
\hline
\end{tabular}

Tableau 5. - Réalisation multimodale de la cohésion discursive. 
Il ressort que les gestes cohésifs de structuration et référentiels sont à la fois complémentaires et indispensables à la cohésion discursive, car si les uns assurent la segmentation du discours en unités, les autres assurent l'intégration de ces unités au niveau textuel. D'un point de vue plurifonctionnel, si les gestes référentiels construisent des référents au niveau de l'énoncé, certains d'entre eux s'attachent à maintenir et assurer, au niveau textuel, la continuité référentielle. En parallèle, si les gestes de structuration accentuent des syllabes ou des mots (i.e. mis en relief de certains par rapport à d'autres) au niveau de l'énoncé, certains d'entre eux accentuent des unités beaucoup plus importantes : thème de l'histoire, épisode, acte narratif, au niveau textuel.

\section{Remarques conclusives}

Au terme de ce travail, on peut faire trois remarques : d'abord, d'un point de vue développemental, les enfants de 9-10 ans ont acquis la plupart des outils textuels et maitrisent d'ores et déjà les rudiments de la narration. Ils sont capables de produire un récit cohésif, avec un début descriptif, un enchainement actionnel et une fin conclusive.

Ensuite, d'un point de vue multimodal et sémiotique, l'étude de la gestualité enfantine nous a permis de montrer que la cohésion discursive n'est pas exclusivement réservée aux seuls moyens linguistiques; certains gestes coverbaux peuvent soutenir le message verbal, sinon s'y substituer, pour remplir cette fonction. De plus, la gestualité cohésive est suffisamment flexible pour s'occuper aussi bien du produit de la narration (la continuité référentielle) que de la gestion de ce produit (la structuration de l'activité narrative).

Enfin, d'un point de vue didactique, ce qui est vrai pour le langage oral peut l'être, en partie, pour le langage écrit, puisque, au-delà des divergences qui séparent les deux modalités (oral et écrit), la capacité à produire du langage organisé en discours est une capacité générale et non une capacité propre à l'oral, même si elle s'actualise d'abord en parole (à l'oral) puis à l'écrit. Des données complémentaires que nous analysons actuellement : comparaison sémiotique des récits orauxécrits, devraient nous fournir des enseignements riches pour la didactique de l'écrit; si la capacité à produire du langage textuellement organisé émerge plus tôt dans les conduites de parole, on voit l'intérêt que présente le fait de solliciter ce type de production chez l'enfant : cela revient à l'entrainer à la production de textes écrits via les textes oraux, 
ce qui induit, du même coup, une remise en question de certaines activités pédagogiques.

\section{RÉFÉRENCES BIBLIOGRAPHIQUES}

CAlbris G. (1997) : «Multicanalité de la communication et multifonctionnalité du geste», dans J. Perrot (coord.), Polyphonie pour Yvan Fonag, Paris, L'Harmattan, p. 61-70.

CAlbris G. (2002) : «Sémantisme des connecteurs : nuancement du verbal par le gestuel», Lidil, n 26, «Gestualité et syntaxe», p. 139-153.

Colletta J.-M. (2004) : Le développement de la parole chez l'enfant âgé de 6 à 11 ans, Hayen (Belgique), Mardaga.

Colletta J.-M., Kunene R., Venouil A., Kaufmann V. et Simon J.-P. (2009) : «Multitrack annotation of child language and gestures», dans M. Kipp, J.-C. Martin, P. Paggio et D. Heylen (éds), Multimodal Corpora: From models of natural interaction to systems and applications, Berlin, Springer-Verlag, LNAI 5509, p. 54-72.

Colletta J.-M., PellenQ C., Guidetti M. (2010) : «Age-related changes in co-speech gesture and narrative: Evidence from French children and adults », Speech Communication, vol. 52, nº 6, p. 565-576. [doi:10.1016/ j.specom.2010.02.009].

Cosnier J. et Brossard A. (1984) : La communication non verbale, Neuchâtel / Paris, Delachaux et Niestlé.

Cosnier J. et Vaysse J. (1997) : «Sémiotique des gestes communicatifs », Nouveaux actes sémiotiques, $\mathrm{n}^{\circ}$ 52, p. 7-28.

Fantazi D. et Colletta J.-M. (2010) : «L'effet du contenu narratif sur la focalisation dans les gestes iconiques », Actes publiés des $X X V I I I^{e s}$ Journées d'étude sur la parole (JEP'10) [Mons, Belgique, 25-28 mai 2010], p. 89-92. Disponible sur <http://w3.umh.ac.be/jep2010/partenaires.php>.

Gullberg M., De Bot K. et Volterra V. (2008) : «Gestures and some key issues in the study of language development», Gesture, vol. 8, n 2, p. 149-179.

Kendon A. (1972): «Some relationships between body motion and speech», dans A. W. Siegman et B. Pope (éds), Studies in dyadic communication, Elmsford, Pergamon Press, p. 177-210.

KitA S. et ÖzyüreK A. (2007) : «How does spoken language shape iconic gestures?», dans S. Duncan, J. Cassell et E. T. Levy (éds), Gesture and the Dynamic Dimension of Language: Essays in Honor of David McNeill, Amsterdam, John Benjamins, p. 67-74.

LABOv W. (1978) : Le parler ordinaire, Paris, Minuit. 
MCNeILL D. (1992): Hand and mind: What gestures reveal about thought, Chicago, University of Chicago Press.

McNeill D. (2000) : Language and gesture, Cambridge, Cambridge University Press.

SCHERER K. R. (1984) : «Les fonctions des signes non verbaux dans la conversation », dans J. Cosnier et A. Brossard (dir.), La communication non verbale, Neuchâtel, Delachaux et Niestlé, p. 71-100. 